\title{
Sense and nonsense in thinking about anomaly and metaphor
}

\author{
HOWARD R. POLLIO and MICHAEL K. SMITH \\ University of Tennessee, Knoxville, Tennessee 37916
}

\begin{abstract}
In an attempt to determine how subjects dealt with four types of logical sentence relationships when a figurative possibility was present, two different experiments were run using a procedure originally developed by Steinberg (1970a). Results indicated that a great many anomalous sentences are readily classified as metaphoric and that individual differences play an important role in the use of these categories. Further analyses revealed that sentences having the order "The 'human' is an 'animal" " were more easily classified as metaphorical than sentences having any other order in the present set of sentences. The findings were discussed in terms of their implications for semantic theory, where it was noted that metaphoric diction makes a purely logical analysis of language exceedingly suspect and that any attempt to define sentence universals must include not only figurative language, but individual differences as well.
\end{abstract}

When is anomaly anomalous and when is it metaphoric? Although this question may appear a bit frivolous, it is clear that any theory of metaphor must contend with anomaly and that any analysis of anomaly must contend with metaphor. One strategy for dealing with this relationship has been to ignore it. For example, Steinberg $(1970 \mathrm{a}, 1970 \mathrm{~b}, 1975)$ tested the ability of college age subjects to code sentences into four categories considered fundamental to natural language use: synthetic, analytic, contradictory, and anomalous. Basing his work on Katz's (1967) suggestion that such distinctions are meaningful to adult speakers, Steinberg showed that subjects successfully coded sentences into one of these four types as long as they used "only the literal meanings of the words and not any metaphorical meanings" (1970a, p. 39). On the basis of these results, Steinberg wrote sentence interpretation rules that described, from the interaction of logical semantic features, how specific sentences fell into each of the major sentence categories.

The larger question raised by ruling out the possibility of metaphoric diction is perhaps best phrased as follows: Are logically anomalous sentences invariably nonsensical, or are there conditions under which they can be meaningfully interpretable? A recent experiment dealing with this issue (Pollio \& Burns, 1977) showed that many randomly generated word sets could be interpreted metaphorically and that subjects asked to learn such "sentences" did significantly better if they had interpreted the sentences before, rather than after, learning. These results suggest that speakers are able to interpret almost any grammatically correct word string and that semantic theories must take this into account.

Basic, then, to any theory of semantics and language use must be some notion as to how ordinary speakers deal with anomaly and metaphor. Specifically, in the case of Steinberg's experiment, it seems reasonable to ask what would happen if subjects were given an option to code sentences into the category of metaphoric as well as into the categories of synthetic, analytic, contradictory, and anomalous. Under these conditions, could a feature theory such as that proposed by Steinberg describe the resulting pattern of categorizations, and would the results of such an experiment have any bearing on the relationships of anomaly to metaphor and vice versa? The present set of studies explored these questions within the context of the sentence categorization task orginally devised by Steinberg.

In order to examine the effects of introducing an additional category into the Steinberg procedure, two experiments were run. Experiment 1 was an exact replication of Steinberg's (1970a) original four-category experiment. Experiment 2 used the same Steinberg procedure except that subjects were allowed to code sentences into five rather than four different categories.

\section{METHOD}

\section{Subjects}

The subjects were 40 college students, mostly freshmen, who were enrolled in introductory psychology courses at the University of Tennessee. Twenty subjects were used in Experiment 1, the replication study, and 20 in Experiment 2. Both male and female subjects were used, and all subjects received course credit for their participation.

\section{Materials}

In Steinberg's original experiment, as well as in our first study, subjects were presented with five examples of four different types of sentences: Type 1 sentences were described as synthetic; Type 2, analytic or redundant; Type 3, contradictory; Type 4, anomalous. After studying these examples and jotting down a brief description of each type of sentence, subjects were asked to categorize a new set of sentences into one of the four categories. 
In our second experiment, subjects were presented with an instruction booklet containing five examples of five different sentence types. Sentences used as examples for Types 1 through 4 were taken from Steinberg's original study, whereas sentences used to exemplify Type 5 were specifically developed for use in the present study.

For example, a Type 1 (synthetic) sentence was, The dog is a poodle; Type 2 (analytic), The tulip is a flower; Type 3 (contradictory), The dog is a cat; Type 4 (anomalous), The mountain is a frog.

In Experiment 1, the Steinberg replication study, the instruction booklet contained only examples of these four sentence types. The specific Type 5, or metaphoric, sentences in the instruction booklet presented to the subjects in Experiment 2 were: The car is a lemon. The woman is a rose. The man is a wolf. The job is a snap. The mind is a mirror.

As can be seen, Type 5 sentences were restricted primarily to examples of cliched usage. Only the last item seems to present a sentence that might in any way be considered as novel figurative usage.

\section{Procedure}

The instructions given to all subjects were as follows: "Please study the above [four] five sets of sentences presented on page one and try to write a few words describing each type of sentence. This description should be written in the space provided below each set of examples. Describe these sentences in whatever words you feel comfortable using." Then subjects were asked to code all sentences into one of the (four) five types previously explained.

\section{Materials}

All sentences used in this experiment were taken from Steinberg's original study and were of the form: The

a inserted into the blank sentence frame presented above: man, woman, person, husband, wife, spouse, fiance, fiancee, betrothed, ram, ewe, sheep, and chair. All items were originally selected by Steinberg so as to represent the semantic dimensions of ANIMATENESS, HUMANNESS, SEX, MARRIAGE, and ENGAGEMENT. As in Steinberg's study, unusual items such as fiance, fiancee, ewe, spouse, and betrothed were glossed in the instructions.

All possible pairs of words were used within the same sentence frame, except for sentences in which both items were the same. For the present set of words this procedure yielded a total of 156 sentences. The sentences were randomized into a small booklet and presented to the subjects for coding into one of the four (five) sentence types. Any subject who felt none of the categories was appropriate was asked to write "None" in the answer blank. Each subject saw the sentences in a different random order and all subjects rated all sentences. For most subjects the task took approximately $1 \mathrm{~h}$; no specific time limit, however, was set.

\section{RESULTS}

Table 1 presents the percent of correct responses for each of the four types of sentences, according to the scheme initially used by Steinberg, for both Experiments 1 and 2. For purposes of comparison, Steinberg's results are also presented in Table 1. There are large differences between the groups for all sentence types, with Steinberg's subjects always producing higher correct placement values (except for Experiment 1, Type 4). In order to evaluate the significance of these differences, $t$ tests were computed using Steinberg's mean percentage as a null hypothesis. Results of the analysis showed that differences were significant for two of the comparisons in the replication group and for all four comparisons in Experiment 2. The largest differences between the sets of data for both groups concerned contradictory sentences. In the replication experiment, only two sentence types showed a percent correct categorization value equal to that found in Steinberg's original study. In Experiment 2, the introduction of a Type 5 response served to change the proportion of correct placements not only for Type 4 sentences but also for all other categories, with a particularly strong effect on Type 3 (contradictory) sentences.

Since adding the option of a Type 5 response produced marked differences in how sentences were categorized, it seemed important to determine into which categories logically incorrect responses were coded. Table 2 presents the distribution of sentence placements for all 20 subjects in Experiment 2 as a consequence of logical type: Diagonal entries represent correct responses, while off-diagonal entries represent incorrect placements.

From these results, it is clear that, if a Type 1 sentence was confused with any other type of sentence, it was usually with a Type 2 sentence (about $25 \%$ of the time). Similarly, Type 2 sentences were most often confused with Type 1 sentences (20\%). Surprisingly, Type 3 sentences were confused with several different sentence types at a $3 \%$ to $16 \%$ rate. These sentences were coded into the "None" category about $10 \%$ of the time and Type 5 was least often used as a coding for a Type 3 sentence. Although Type 4 sentences were correctly placed only $59 \%$ of the time, present results showed that $20 \%$ of incorrect placements were made into Type 5. For Type 4 sentences, $79 \%$ of all responses placed them into the two categories of anomaly and metaphor even though there was no systematic placement for the remaining $21 \%$ of the judgments. Type 5 was seldom used to categorize any of the other three types of sentences.

Table 1

Percentage Correct Category Placements for Subjects in Two Experiments

\begin{tabular}{|c|c|c|c|c|c|c|c|}
\hline Category & Type & $\begin{array}{c}\text { Total } \\
\text { Sentences } \\
156 \\
\end{array}$ & $\begin{array}{c}\text { Steinberg } \\
(1970 a) \\
N=28\end{array}$ & $\begin{array}{c}\text { Experiment } 1 \\
\text { (Replication) } \\
\mathrm{N}=20\end{array}$ & Diff. & $\begin{array}{c}\text { Experiment } 2 \\
\mathrm{~N}=20\end{array}$ & Diff. \\
\hline Synthetic, or Informative & 1 & 26 & 82.4 & 79.0 & 3.4 & 65.0 & $17.4^{*}$ \\
\hline Analytic, or Redundant & 2 & 18 & 87.0 & 74.0 & $13.0^{*}$ & 70.0 & $17.0^{*}$ \\
\hline Contradictory & 3 & 34 & 75.0 & 57.0 & $18.0^{*}$ & 49.1 & $25.9 *$ \\
\hline Amphigorous & 4 & 78 & 79.9 & 85.0 & 5.1 & 59.1 & $20.8 *$ \\
\hline
\end{tabular}

${ }^{*} p<.05$ 
Table 2

Matrix of Sentence Placement, Experiment 2

\begin{tabular}{lrrrrrrr}
\hline \multirow{2}{*}{$\begin{array}{c}\text { Logical } \\
\text { Type }\end{array}$} & $\begin{array}{r}\text { Total } \\
\text { Items }\end{array}$ & \multicolumn{1}{c}{1} & \multicolumn{6}{c}{ Subject Placements (Percent) } \\
\cline { 3 - 9 } & 520 & 65 & 25 & 5 & 1 & 2 & 2 \\
\hline 1. Synthetic & 560 & 20 & 70 & 4 & 2 & 3 & 1 \\
2. Analytic & 360 & 16 & 12 & 49 & 10 & 3 & 10 \\
3. Contradictory & 680 & 3 & 4 & 59 & 20 & 8 \\
4. Anomalous & 1560 & 6 & 3 & 4 & $50 n e$ \\
Total Items & 3120 & 601 & 515 & 434 & 1008 & 346 & 216 \\
\hline
\end{tabular}

\section{Individual Differences in Using Type 5}

The introduction of a Type 5, or metaphoric, category produced a marked effect on the logically proper classification of sentences. One question that arises, however, is whether such changes were the result of individual judgments or if they represent a general trend for all subjects. To answer this question, the number of Type 5 responses for each of the 20 subjects was tabulated. From these data, three distinct groups were readily identifiable. One group of six subjects produced very few Type 5 responses (between 0 and 2 for all 156 sentences); a second group of seven subjects produced a large number of Type 5 responses (more than 29 such judgments); a third group of seven subjects produced between 9 and 20 Type 5 responses. There, thus, appeared to be sharp differences between subjects in the first group, who seldom, if ever, categorized sentences as Type 5, and subjects in the second group, who categorized up to $25 \%(39 / 156)$ of their sentences as Type 5 . In further discussion, the first group is termed the literal group, while the second is referred to as the figurative group. Subjects showing an intermediate number of Type 5 placements are referred to as the mixed group.

Given the three groups, the question arises as to whether or not they performed differently with respect to the logically correct labeling of the remaining four sentence types when compared to each other and to Steinberg's original subjects. Table 3 presents these comparisons. The literal, mixed, and figurative groups performed more poorly than did Steinberg's subjects. Again, using t tests with Steinberg's mean percentage as a null value, all comparisons were statistically significant except for Types 1,2 , and 4 for the literal group and Types 1 and 2 for the figurative group. Since the number of cases was small, the overall trend is more significant than individual comparisons.

These data further revealed that the literal group performed better than the mixed group, which in turn outperformed the figurative group, on all sentence types, although for Types 1 and 2 the figurative group did slightly better than the mixed group. Subjects who had few Type 5 responses performed almost as well as Steinberg's original subjects (in terms of logically categorizing the sentences). On the other hand, subjects who used a large number of Type 5 categorizations produced fewer logically correct categorizations for each of the remaining four sentence types.

From error matrices produced by the mixed and figurative groups, it was determined that the major source of Type 5 (metaphoric) codings was derived from logically anomalous sentences. Given this result, it seems reasonable to ask if each of the 78 anomalous sentences was equally likely to be coded as metaphoric. A breakdown of all anomalous sentences that were categorized by subjects as metaphoric revealed that sentences of the order The (human) is an (animal) accounted for $70 \%$ of the logically anomalous sentences labeled as metaphoric. Conversely, sentences such as The (animal) is a (human) were judged as metaphoric only $20 \%$ of the time. All other sentence frames [The (human) is a (inanimate object), The (inanimate object) is a (human), etc.] accounted for fewer than $6 \%$ of the Type 5 judgments, leaving the distinct impression that sentences comparing a human to an animal were often, and easily, seen as metaphoric. Sentences in which an animal is seen as a human were much less often coded as metaphoric within the context of the present set of sentences, although both possibilities regularly occur in the figurative category of anthropomorphic metaphor.

\section{DISCUSSION}

Providing subjects with the possibility of coding the set of rather uninteresting sentences into a metaphoric category produced a number of interesting consequences. (1) It reduced the number of sentences subjects were willing to code as anomalous; (2) it reduced the number of logically correct codings for sentences not necessarily having anything to do with anomaly and/ or metaphor; (3) it revealed surprisingly large individual differences in a given speaker's willingness to use metaphoric diction. Finally, the results revealed that word order and feature conflict (human vs. animal) were crucial in determining whether or not a given pair of words was seen in a metaphoric or anomalous relationship.

Thus, under conditions of Experiment 2, subjects often classified human/animal conflicts as metaphoric rather than as anomalous. This was in contrast to a strictly logical analysis of the featural relationship existing between the two nouns comprising the sentence. Experiment 2 also showed strong individual differences in a given subject's tendency to place sentences into the Type 5 category. Such differences suggest that the perception of anomaly (and metaphor) is highly dependent on individual judgments and cast doubt on the possibility of developing a single, logical definition of anomaly (or metaphor) that

Table 3

Percentage Correct Category Placements for Experiment 2 by Group

\begin{tabular}{ccccc}
\hline $\begin{array}{c}\text { Category } \\
\text { Type }\end{array}$ & $\begin{array}{c}\text { Steinberg } \\
\mathrm{N}=28\end{array}$ & $\begin{array}{c}\text { Figurative } \\
\mathrm{N}=7\end{array}$ & $\begin{array}{c}\text { Mixed } \\
\mathrm{N}=7\end{array}$ & $\begin{array}{c}\text { Literal } \\
\mathrm{N}=6\end{array}$ \\
\hline 1 & 82 & 62 & $61^{*}$ & 72 \\
2 & 87 & 71 & $67^{*}$ & 73 \\
3 & 75 & $43^{*}$ & $51^{*}$ & $52^{*}$ \\
4 & 80 & $50^{*}$ & $55^{*}$ & 75 \\
\hline
\end{tabular}

${ }^{*} p<.05$. 
would be valid for all subjects under all (or even many) speaking and listening situations.

The results also suggest that sentences judged as metaphoric were usually derived from sentences designed to represent logical anomaly; infrequently, subjects dealt with analytic, synthetic, or, more importantly, with contradictory sentences as examples of metaphor. This finding argues against a number of theoretical analyses in which figurative expression is seen to depend upon additional cognitive processing required to understand sentences originally interpreted as contradictory (Osborne \& Ehninger, 1962; Steinberg, 1975; Thomas, 1969). Only infrequently was logical contradiction coded as figurative.

The introduction of a Type 5 category also made the logically correct placement of sentences in the remaining categories somewhat more difficult. Results presented in Experiment 2 indicated that the simple introduction of a metaphoric possibility decreased the number of logically correct placements for sentence types clearly not metaphoric. In general, where there is metaphor, strong logical analysis is not, and this may explain the the tendency of semantic theorists to exclude metaphor from their theories. A little bit of metaphor greatly damages even the most robust categories of logical analysis.

When individual differences are considered, this conclusion is even more striking. Subjects in the figurative group quite easily classified certain anomalous sentences as metaphoric, while subjects in the literal group were more inclined to disregard metaphor and judged sentences "literally." Such evidence suggests that what is nonsense to one person may make "sense" to someone else and that individual differences must be taken into account in any attempt to develop a list of semantic universals. In fact, the influence of individual differences has been found not only in the present task but in other metaphoric activities as well (Pollio \& Smith, in press), suggesting that such factors must play a key role in any theory of metaphor.

The semantic features approach, initially developed by Chomsky (1965) and carried forward by Katz (1972), occurs not only in Steinberg's work but also in many areas of psychology (see Rosch, 1975; Smith, Shoben, \& Rips, 1974). This class of approach often assumes that lexical knowledge is in some sense logically and hierarchically arranged and that anomaly can be defined in terms of feature conflict. As shown in this study, speakers often were able to derive meaningful interpretations for even the highest level semantic conflict. Furthermore, no logical featural model seems to account for the fact that some individuals chose to interpret sentences as metaphoric, while others did not; nor can it account for the fact that, in certain contexts, even a contradictory sentence such as "The man is a woman" can be understood metaphorically.

The need to question a featural approach to cognitive processes in general, and to metaphor in particular, receives further support from a recent set of studies by Verbrugge and McCarrell (1977). In these studies, recall of metaphoric sentences was cued by a relevant, an irrelevant, or an arbitrary prompt, and results indicated that memory and comprehension of metaphor could not be explained on the basis of preexisting connections between prompts and topics. As Verbrugge and McCarrell put it: "The process of comprehension involves a global transformation of the topic domain ... guided by properties of the vehicle" (p. 518). The impact of individual words cannot be separated from the complete expression under consideration. A sentence such as "The man is a ram" cannot be ruled out as metaphoric simply by virtue of the fact that features appropriate to "man" are incompatible with those evoked by "ram." Metaphor is an active reorganization of words in a sentence or phrase and, as results of the present study indicate, this reorganization depends as much on individual speakers as on semantic interactions within the sentence itself.

\section{REFERENCES}

Сномsкy, N. Aspects of the theory of syntax. Cambridge, Mass: M.I.T. Press, 1965.

KATz, J. J. Some remarks on Quine on analyticity. Journal of Philosophy, 1967, 64, 36-52.

KATZ, J. J. Semantic theory. New York: Harper \& Row, 1972.

Osborne, M. M., \& Ehninger, D. The metaphor in public address. Speech Monographs, 1962, 29, 223-234.

Pollio, H., \& BuRns, B. C. The anomaly of anomaly. Journal of Psycholinguistic Research, 1977, 6, 247-260.

Pollio, H., \& SMith, M. Metaphoric style and complex human problem solving. In R. Hoffman \& R. Honeck (Eds.), The psycholinguistics of figurative language. Hillsdale, N.J: Erlbaum, in press.

Rosch, E. Cognitive representations of semantic categories. Journal of Experimental Psychology: General, 1975, 104, 192-233.

Smith, E., Shoben, E., \& RIPS, L. Structure and process in semantic memory: A featural model for semantic decisions. Psychological Review, 1974, 81, 214-241.

SteinberG, D. D. Analyticity, amphigory and semantic interpretation of sentences. Journal of Verbal Learning and Verbal Behavior, 1970, 9, 37-51. (a)

STEINBERG, D. D. Negation, analyticity, amphigory and the semantic interpretation of sentences. Journal of Experimental Psychology, 1970, 84, 417-423. (b)

STEINBERG, D. D. Semantic universals in sentence processing and interpretation: A study of Chinese, Finnish, Japanese, and Slovenian speakers. Journal of Psycholinguistic Research, 1975, 4, 169-193.

Thомаs, O. Metaphor and related subjects. New York: Random House, 1969.

Verbrugge, R. R., \& McCarRell, N. S. Metaphoric comprehension: Studies in reminding and resembling. Cognitive Psychology, 1977, 9, 494-533.

(Received for publication February 2, 1979.) 